\title{
Primary tumors induce neutrophil extracellular traps with targetable metastasis-promoting effects
}

\author{
Roni F. Rayes, ${ }^{1}$ Jack G. Mouhanna, ${ }^{1}$ loana Nicolau, ${ }^{2}$ France Bourdeau, ${ }^{1}$ Betty Giannias, ${ }^{1}$ \\ Simon Rousseau, ${ }^{3}$ Daniela Quail, ${ }^{4}$ Logan Walsh, ${ }^{4}$ Veena Sangwan, ${ }^{1}$ Nicholas Bertos, ${ }^{1}$ \\ Jonathan Cools-Lartigue, ${ }^{1}$ Lorenzo E. Ferri, ${ }^{1}$ and Jonathan D. Spicer ${ }^{1}$ \\ 'Cancer Research Program and the LD MacLean Surgical Research Laboratories, Department of Surgery, McGill University \\ Health Center (MUHC), Montreal, Québec, Canada. 'Dalla Lana School of Public Health, University of Toronto, Toronto, \\ Ontario, Canada. ${ }^{3}$ Meakins-Christie Laboratories, Department of Medicine, McGill University and the MUHC, Montreal, \\ Québec, Canada. ${ }^{4}$ Goodman Cancer Research Center, McGill University, Montreal, Québec, Canada.
}

\begin{abstract}
Targeting the dynamic tumor immune microenvironment (TIME) can provide effective therapeutic strategies for cancer. Neutrophils are the predominant leukocyte population in mice and humans, and mounting evidence implicates these cells during tumor growth and metastasis. Neutrophil extracellular traps (NETs) are networks of extracellular neutrophil DNA fibers that are capable of binding tumor cells to support metastatic progression. Here, we demonstrate that circulating NET levels are elevated in advanced esophageal, gastric, and lung cancer patients compared with local cancers and healthy controls. Using preclinical murine models of lung and colon cancer, in combination with intravital video microscopy, we show that NETs functionally regulate disease progression and that blocking NETosis through multiple strategies significantly inhibits spontaneous metastasis to the lung and liver. Furthermore, we show how inhibiting tumorinduced NETs decreases cancer cell adhesion to liver sinusoids following intrasplenic injection - a mechanism previously thought to be driven primarily by exogenous stimuli. Thus, in addition to neutrophil abundance, the functional contribution of NETosis within the TIME has critical translational relevance and represents a promising target to impede metastatic dissemination.
\end{abstract}

Authorship note: RFR and JGM share first authorship.

Conflict of interest: The authors have declared that no conflict of interest exists.

Copyright: (c) 2019, American Society for Clinical Investigation.

Submitted: February 6, 2019

Accepted: July 22, 2019

Published: July 25, 2019.

Reference information: JCI Insight. 2019;4(16):e128008

https://doi.org/10.1172/jci.

insight.128008.

\section{Introduction}

Metastasis remains the leading cause of death among cancer patients. The role of the tumor immune microenvironment (TIME) in cancer initiation, progression, and treatment efficacy is well established (1) and has led to the development of cancer immunotherapies $(2,3)$. Granulocytic neutrophils, the most abundant leukocyte in mammals, are the first cells of the innate immune system to be recruited to inflammation sites. They have diverse cytotoxic functions due to high concentrations of proteases and ROS within their granules, which encounter pathogens through phagocytosis, degranulation, or release of neutrophil extracellular traps (NETs). Paralleling a growing appreciation for the link between inflammation and cancer, we $(4,5)$ and others $(6-10)$ have demonstrated a regulatory role for neutrophils during various stages of tumor initiation and metastatic progression.

NETs are neutrophil-derived webs of DNA decorated with granular proteins, including myeloperoxidase (MPO) and neutrophil elastase (NE), that were initially shown to capture and kill foreign pathogens (11-13). However, more recent studies have also shown a noninfectious role for NETosis (14). For example, our group was the first to our knowledge to demonstrate a role for NETs in cancer progression (15). We discovered that NETosis can be induced by sepsis, which in turn promotes metastatic progression by trapping circulating tumor cells and promoting their proliferation in secondary sites $(15,16)$. Additional studies have since reported similar findings, whereby cancer progression is potentiated by NETosis induced by LPS $(17,18)$, tobacco smoke (17), tumor-derived inflammatory cytokines (18-20), or surgical stress (21). These preclinical findings suggest that various types of systemic inflammatory insults may induce NETosis in cancer patients to support advancement of disease. However, data that directly address the relevance of this phenomenon in human cancer patients is absent, and few validated biomarkers exist for the identification of patients who might benefit from NET-directed therapy. 
Evidence for the presence of NETs in humans is focused largely on infection, tissue injury, and autoimmunity, with minimal data in cancer patients. For instance, elevated levels of circulating NETs are observed in blood of patients with inflammatory conditions (22-26) relative to healthy controls. These observations prompt the question of whether tumor-driven inflammation can induce NETs in the absence of infection. Indeed, it is only recently that NETs were thought to be involved in cancer progression and metastasis (reviewed in ref. 27). One group has shown that neutrophils isolated from colorectal cancer patients had increased NET production as compared with neutrophils isolated from age-matched healthy individuals following in vitro stimulation (28). Interestingly, NET levels were shown to be an independent prognostic factor in pancreatic ductal adenocarcinoma (29) and H3 Cittrulline (H3Cit), a good diagnostic and prognostic marker of cancer progression (30), showing the importance of NETs as biomarkers of cancer. Moreover, NETs were shown to facilitate ovarian cancer metastasis to the omentum (31), implicating NETs once again in cancer metastasis.

Our previous work has demonstrated a tumor-promoting role for NETs in preclinical models of cancer $(15,32)$. Here, we investigate whether circulating NET levels are elevated in patients with several high-lethality malignancies, including lung, esophageal, and gastric adenocarcinoma, and we show that higher NET level is associated with disease stage. Using preclinical models of lung and gastrointestinal (GI) cancer, we confirm that tumors prime neutrophils to undergo NETosis in the absence of infection/surgical stress to promote metastatic progression. Further, these protumorigenic NETs can be therapeutically exploited through both pharmacologic and genetic approaches. Importantly, our results show that higher NET levels in cancer patients can be used as a biomarker of progressive disease independent of neutrophil-to-lymphocyte ratio (NLR) or absolute neutrophil count -2 gold-standard clinical biomarkers of cancer prognosis. This study is the one of the first to our knowledge to bring largescale human data to the forefront for several important tumor sites and directly address the relevance of NETosis in human cancer. Therefore, our findings have critical implications for translating NET-directed therapies to clinical trial implementation.

\section{Results}

NET levels correlate with disease stage. To explore whether NETs are present in the circulation in human cancer patients, we used an MPO-dsDNA ELISA (referred to as NET ELISA) (22-24). The sensitivity and specificity of the NET ELISA were confirmed (Supplemental Figure 1, A-E; supplemental material available online with this article; https://doi.org/10.1172/jci.insight.128008DS1). Indeed, stimulating isolated human neutrophils with increasing concentrations of PMA $(0.0625 \mu \mathrm{M}$ to $2 \mu \mathrm{M})$ resulted in a dose-dependent increase in NET levels, as detected by the NET ELISA (Supplemental Figure 1A). Sytox staining of those stimulated neutrophils confirmed that what the assay measures are NETs, as seen using a fluorescent plate reader (Supplemental Figure 1B) and under a fluorescent microscope (Supplemental Figure 1C). In addition, we show that the ELISA is specific to NETs and does not measure any neutrophil or tumor DNA (Supplemental Figure 1D). Moreover, we show that NETs can be measured in plasma or serum from whole blood and that an increase in NETs following PMA stimulation or a decrease in NETs following DNase1 treatment can be detected by the NET ELISA (Supplemental Figure 1E). Following this validation, we measured circulating NET levels in the plasma of 60 treatment-naive lung or upper GI (esophagogastric) adenocarcinoma patients compared with 15 healthy individuals. We observed a trend of higher level of circulating NETs in cancer patients with advanced cancer (stage III and -IV esophagogastric and stage II and -III lung) compared with healthy controls $(P=0.0265)$ and significantly higher NET levels in patients with advanced cancer (stage III and -IV esophagogastric and stage II and -III lung) compared with patients with local disease (stage I and -II esophagogastric and stage I lung) $(P=0.009$, Figure 1A; cohort demographics are presented in Table 1). There was no difference in NET levels between patients with local disease and healthy controls $(P=0.20$, Figure $1 \mathrm{~A})$. Within the esophagogastric patients, NET levels were higher in patients with overall stage III and -IV disease compared with stage I and -II ( $P=0.03$, Figure 1B), T3 and T4 tumors compared with T1 and T2 (Supplemental Figure 2A), positive lymph node status compared with no lymph node involvement (Supplemental Figure 2B), and distant metastasis compared with nonmetastatic tumors (Supplemental Figure 2C). Within the lung patients, NET levels were higher in patients with overall stage II and -III disease compared with stage I $(P=0.05$, Figure $1 C)$ and $\mathrm{T}^{+}$tumors compared with T1 (Supplemental Figure 2D). Together, these data indicate that treatment and surgical stress are not required to induce NETosis in cancer patients (21) and that the presence of a tumor alone correlates with an increase in NET levels, suggesting that tumors can induce NETosis in the absence of any other stimuli. 
A Cancer patients vs. healthy volunteers

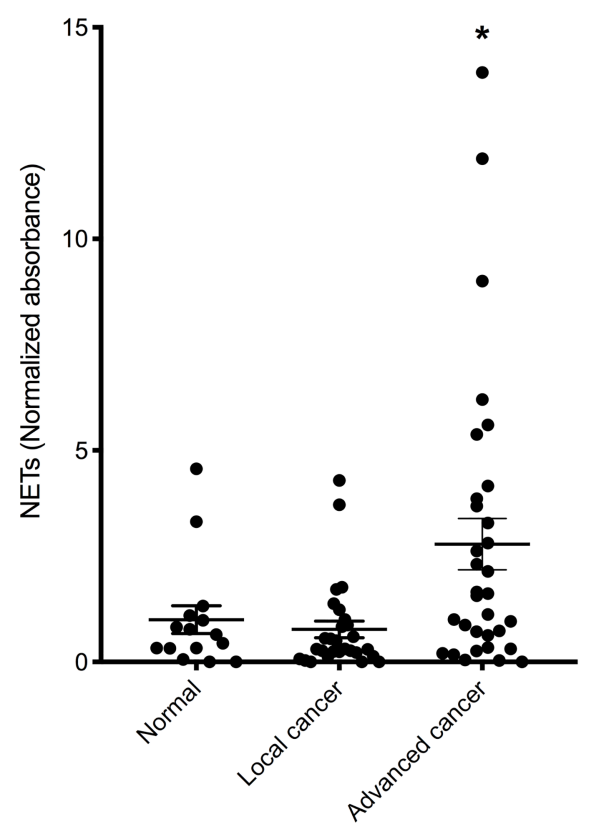

B Esophagogastric adenocarcinoma

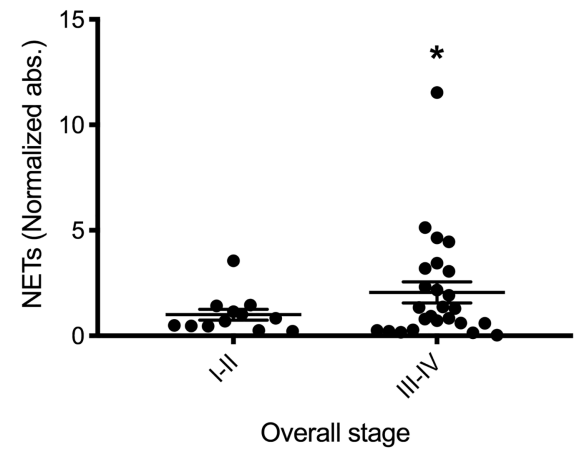

C

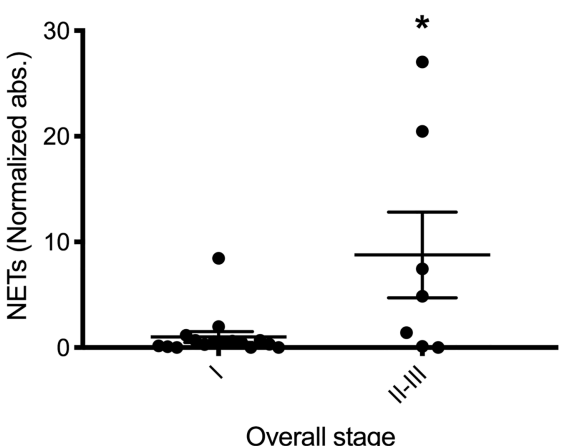

Figure 1. Circulating NET levels in esophagogastric and lung adenocarcinoma patients. (A) NET levels (normalized to the average NET level of healthy individuals, labeled as normal) obtained by the NETs ELISA are shown for patients with local cancer $(n=28)$, compared with both patients with advanced cancer $(n=32)$ and with healthy individuals (labeled as normal; $n=15$ ). A Kruskal-Wallis test was used to calculate significance because the data were not normally distributed, as assessed by the Kolmogorov-Smirnov test. (B) NET levels (normalized to the average NET level of overall stage I and -II) for esophagogastric adenocarcinoma patients are shown for overall stage I and -II $(n=12)$ and stage III and -IV $(n=25)$. (C) NET levels (normalized to the average NET level of overall stage I) for lung adenocarcinoma patients shown plotted for overall stage I $(n=16)$ and stage II and -III $(n=7)$. A Student $t$ test was used to assess statistical significance for $\mathbf{B}$ and $\mathbf{C}$. Mean \pm SEM is shown for all panels. ${ }^{*} P<0.05$.

Advanced cancer stage and diabetes are independent predictors of NET levels. We next wanted to determine whether NET levels could be used as biomarkers of cancer stage. Multivariable logistic regression analyses were performed on the cancer patient cohort to identify whether stage was an independent predictor of NET levels, adjusting for the following confounders: age, sex, BMI, comorbidity, smoking, and diabetes. The analyses revealed that cancer stage and diabetes are significant independent predictors of NET levels (Table 2). Moreover, while NET levels correlated with overall stage in both esophagogastric (Figure 1B) and lung (Figure 1C) patients, NLRs did not correlate with overall stage in either patient group (Supplemental Figure 3, A and C). NLRs did, however, correlate with T staging in both groups (Supplemental Figure 3, B and D). Similarly, absolute neutrophil counts did not correlate with overall stage (Supplemental Figure 4, A and C) but did correlate with T staging (Supplemental Figure 4, B and D). Finally, neither NLR nor absolute neutrophil count correlated with NET levels (Supplemental Figure 3E and Supplemental Figure 4E); however, as expected, absolute neutrophil count correlated with NLR (Supplemental Figure 4F). These findings indicate that NET levels are strong prognostic factors of advanced-stage cancer independent of NLR and neutrophil count. Therefore, NET levels may represent a more sensitive measure of cancer-related inflammation than more crude measures, such as NLR, that have been investigated extensively in the clinical literature.

NET levels are correlated with the presence and progression of primary lung tumors in preclinical models. To better delineate the functional contribution of NETs during cancer progression, we used immunocompetent syngeneic murine models of lung and GI cancer. We injected Lewis-lung carcinoma cells (H59) s.c. into the flank of C57BL/6 mice. We collected plasma from the peripheral blood of H59 tumor-bearing mice (TBM) 1-2 weeks and 3-4 weeks after tumor inoculation and compared the NET 
Table 1. Demographics and clinical characteristics of cancer patients and healthy individuals

\begin{tabular}{|c|c|c|c|}
\hline & $\begin{array}{l}\text { Patients with esophagogastric } \\
\text { adenocarcinoma }(n=37)\end{array}$ & $\begin{array}{l}\text { Patients with lung adenocarcinoma } \\
\qquad(n=23)\end{array}$ & Healthy individuals ( $n=15)$ \\
\hline \multicolumn{4}{|l|}{ Sex } \\
\hline Male & 25 & 15 & 8 \\
\hline Female & 12 & 8 & 7 \\
\hline \multicolumn{4}{|l|}{ Age } \\
\hline Mean \pm SEM & $68 \pm 2$ & $69 \pm 2$ & $31 \pm 3$ \\
\hline Range & $39-94$ & $49-85$ & $23-53$ \\
\hline \multicolumn{4}{|l|}{ Overall stage } \\
\hline I & 5 & 16 & - \\
\hline II & 7 & 4 & - \\
\hline III & 17 & 3 & - \\
\hline IV & 8 & 0 & - \\
\hline \multicolumn{4}{|l|}{ T stage } \\
\hline $\mathrm{T} 1$ & 5 & 9 & - \\
\hline $\mathrm{T} 2$ & 2 & 8 & - \\
\hline T3 & 22 & 5 & - \\
\hline T4 & 8 & 1 & - \\
\hline \multicolumn{4}{|c|}{ Lymph node status (N stage) } \\
\hline NO & 14 & 21 & - \\
\hline $\mathrm{N}+$ & 23 & 2 & - \\
\hline \multicolumn{4}{|c|}{ Distant metastasis (M stage) } \\
\hline MO & 29 & 23 & - \\
\hline M1 & 8 & 0 & - \\
\hline \multicolumn{4}{|l|}{ BMI } \\
\hline Mean \pm SEM & $27 \pm 5$ & $26 \pm 5$ & - \\
\hline \multicolumn{4}{|l|}{ Smoking status } \\
\hline Ever smoked & 15 & 18 & - \\
\hline \multicolumn{4}{|c|}{ Charlson Comorbidity Index } \\
\hline 1 & 1 & 1 & - \\
\hline 2 & 9 & 3 & - \\
\hline 3 & 6 & 3 & - \\
\hline 4 & 10 & 6 & - \\
\hline 5 & 4 & 5 & - \\
\hline 6 & 6 & 4 & - \\
\hline 7 & 1 & 1 & - \\
\hline \multicolumn{4}{|l|}{ Diabetes } \\
\hline Diabetic & 7 & 5 & - \\
\hline
\end{tabular}

levels with those in non-TBM (Figure 2A). We observed significantly higher circulating NET levels 3-4 weeks after tumor injection compared with either non-TBM or to TBM 1-2 weeks after tumor injection (Figure $2 \mathrm{~B}$ ). Once the tumor reached approximately $1.5 \mathrm{~cm}^{3}$, it was resected, and NET levels were assessed. We observed that, within 2 days following tumor resection and up to the time of sacrifice (2 weeks after resection), NET levels fell back to their baseline levels prior to tumor inoculation (Figure 2B). These data recapitulate our findings in patients, by demonstrating that NET levels are higher in the presence of a tumor compared with non-tumor-bearing controls.

NET levels in TBM are decreased by DNase1 treatment, by NE inhibition, and in Pad4 ${ }^{-1-}$ mice. Next, we sought to assess the functional contribution of NETs to tumor progression by treating tumor bearing mice with a NET degrading enzyme (DNase1) or a NET inhibitor (NEi, Sivelestat). We measured NET levels in these 2 treatment groups of mice 3-4 weeks after H59-GFP lung tumor inoculation, when the tumor reached approximately $2 \mathrm{~cm}^{3}$ and compared them with untreated TBM (Figure 2C). TBM treated with DNase1 or NEi had significantly lower NET levels compared with untreated TBM (Figure 2D). These data suggest that NET-targeted therapies using a NET degrader, DNase1, or a NET inhibitor, NEi, can decrease circulating NET levels. 
Table 2. Multivariable logistic regression models to predict NET levels

\begin{tabular}{lcc}
\hline Variable & \multicolumn{1}{c}{ Multivariable model } \\
& OR (95\% CI) & $P$ value \\
Stage & $0.138(0.022-0.254)$ & 0.020 \\
Age & $-0.0015(-0.011-0.008)$ & 0.76 \\
Sex (male vs. female) & $0.098(-0.025-0.221)$ & 0.12 \\
BMI & $-0.012(-0.026-0.001)$ & 0.07 \\
Charlson Comorbidity Index & $-0.034(-0.114-0.046)$ & 0.405 \\
Smoking (ever vs. never) & $-0.046(-0.162-0.070)$ & 0.436 \\
Diabetes & $0.313(0.148-0.479)$ & 0.001
\end{tabular}

We also utilized genetically modified mice that lack peptidyl arginine deiminase type IV (PAD4), a key enzyme in NET formation (33), and thus are NET-deficient. PAD4 is an important mediator of innate immunity since $P A D 4^{-/-}$mice were shown to be more vulnerable to bacterial infection (34). Moreover, it was recently shown that many known physiological NET inducers (N-Formylmethionyl-leucyl-phenylalanine [fMLP], GM-CSF, TNF $\alpha$, or PMA) are PAD4 dependent (35). Here again, we found that PAD4 $4^{-1-}$ TBM had significantly lower NET levels compared with untreated TBM (Figure 2D). Therefore, primary tumors induce NET release in a PAD4-dependent manner, and PAD4-targeted therapies using PAD4 inhibitors can decrease circulating, tumor-induced NET levels.

Neutrophils are more sensitized to NETose in TBM compared with NET-deficient mice. To assess whether neutrophils are more primed toward NETosis in the presence of cancer, we used imaging flow cytometry, which is a potentially novel flow-based imaging technique to measure the nucleus size of circulating neutrophils as a surrogate for their activation state (36). A seminal event in NETosis is the decondensation of DNA prior to its release, resulting in a measurable increase in the size of the nucleus (36). We first validated this assay using isolated murine neutrophils stimulated with PMA compared with nonstimulated neutrophils. By quantifying the change in nuclear area in images of circulating neutrophils, we were able to infer the activation state of neutrophils (Supplemental Figure 5A and Supplemental Figure 3A). We observed that the median baseline nucleus area of neutrophils in TBM prior to stimulation $\left(87 \pm 3 \mu \mathrm{m}^{2}\right)$ was not significantly different compared with neutrophils from non-TBM $\left(88 \pm 3 \mu \mathrm{m}^{2}\right)$, NEi-treated TBM $\left(87 \pm 1 \mu \mathrm{m}^{2}\right)$, DNase1-treated TBM $\left(84 \pm 2 \mu \mathrm{m}^{2}\right)$, and PAD4 $4^{--} \mathrm{TBM}\left(89 \pm 4 \mu \mathrm{m}^{2}\right)$ (orange curves in Figure $3 C$; population selection is shown in Supplemental Figure $5 \mathrm{~B})$. However, neutrophils from TBM were more sensitive to PMA stimulation (500 nM, 1 hour; $10 \% \pm 2 \%$ increase in nucleus size) than non-TBM $(3 \% \pm$ $1 \%$ increase). Blockade of NET formation in NEi-treated TBM ( $3 \% \pm 1 \%$ increase) and PAD4 $4^{-1-}$ TBM ( $5 \%$ $\pm 1 \%$ increase) rescues the phenotype (Figure 3, A-D). DNase1-treated TBM were not less sensitive than TBM $(11 \% \pm 1 \%$ increase; Figure 3, B-D), a likely explanation being that DNase1 degrades NETs after they are produced and has no effect on nuclear decondensation. These data suggest that primary tumors prime circulating neutrophils to release NETs and that NET inhibitors rescue this phenotype.

NET-deficient mice have reduced cancer cell adhesion to the liver. Previous studies have shown that neutrophils travel to the liver sinusoids and release NETs to capture bacteria from the bloodstream during sepsis (37). Given our previous findings that NETs facilitate liver metastasis (15), we next asked whether NETs were similarly capable of capturing cancer cells from circulation to facilitate liver metastasis in the absence of sepsis. We performed intravital microscopy (IVM) to measure in vivo adhesion of intrasplenically injected H59-GFP cells in TBM. We observed a significant increase in in vivo hepatic adhesion of intrasplenically injected cells in TBM compared with non-TBM, DNase1- or NEi-treated TBM, and PAD4 $4^{--}$TBM (Figure 4, A-C). Since our initial analysis included patients with both lung and GI cancers (Figure 1), as a complementary model, we also performed intrasplenic injection of the colon cancer cell line MC38-RFP. We similarly observed a significant increase in in vivo hepatic adhesion of intrasplenically injected MC38-RFP in TBM compared with non-TBM, DNase1or NEi-treated TBM, and $P A D 4^{-1-}$ TBM (Figure 4, D and E). These data demonstrate that both lung and colon primary tumors induce NETs that promote adhesion of circulating tumor cells to the liver, even in the absence of sepsis. 
A

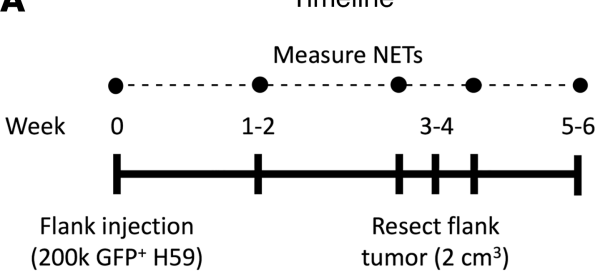

B

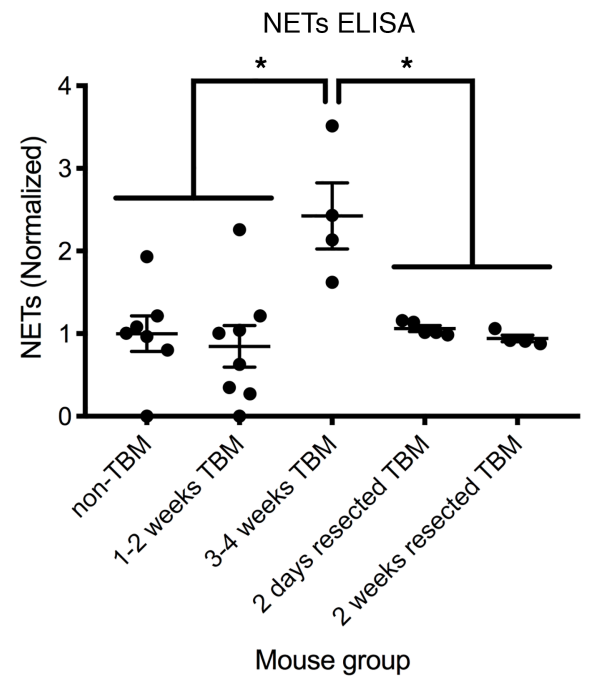

C

Timeline

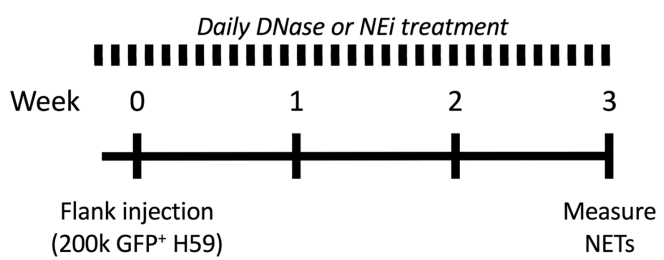

D

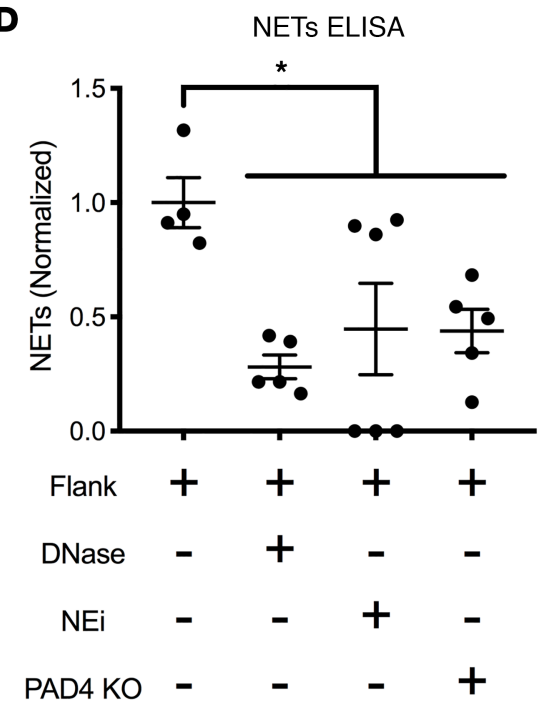

Figure 2. Circulating NET levels are decreased in tumor-bearing mice (TBM) following tumor resection or treatment with a NET inhibitor or degrader and in PAD4 ${ }^{-/-}$TBM with inhibited NET formation. (A) Timeline of murine lung carcinoma NET measurements. (B) Mean ( \pm SEM) NET levels (normalized to non-TBM) are shown for non-TBM $(n=7)$, TBM $1-2$ weeks $(n=8)$ and 3-4 weeks $(n=4)$ after tumor inoculation, as well as TBM 2 days $(n=5)$ and 2 weeks $(n=4)$ after resection. (C) Timeline of the murine lung carcinoma NET measurements. (D) Mean ( \pm SEM) NET levels (normalized to TBM) are shown for TBM $(n=4)$, TBM treated with DNase1 $(n=5)$ or NEi $(n=6)$, and PAD4 ${ }^{-1-}$ TBM $(n=5)$. One-way ANOVA was used to assess statistical significance for $\mathbf{B}$ and $\mathbf{D}$. ${ }^{*} P<0.05$.

NET-deficient mice have reduced spontaneous liver and lung metastasis. To assess whether the tumor-induced NETs affect tumor cell metastasis, we injected s.c. into the flank H59-GFP cells that have been previously shown to spontaneously metastasize to the lung and liver (38), two of the most common metastatic sites. To visualize liver and lung metastases in H59-GFP TBM, the primary tumor was resected when it reached approximately $1.5-2 \mathrm{~cm}^{3}$, and mice were sacrificed after 2 weeks (Figure $5 \mathrm{~A}$ ). Numerous lung metastases were observed (>300 tumor cells per lung) and were, thus, quantified by extracting and homogenizing the lung tissue and counting $\mathrm{GFP}^{+}$tumor cells by flow cytometry. There was a marked reduction in spontaneous lung metastasis in the NET-deficient TBM, DNase1- or NEi-treated TBM, and PAD4 $4^{-1}$ TBM, compared with untreated TBM (Figure 5, B and C, and Supplemental Figure 6).

To assess spontaneous liver metastasis formation, murine livers were extracted, and liver metastases were quantified using fluorescence microscopy once the flank lesions reached approximately $1.5-2 \mathrm{~cm}^{3}$ (Figure 5D). Flow cytometry was not needed in this case, as fewer cells metastasized to the liver as compared with lungs. We observed reduced spontaneous liver metastasis in NET-deficient tumor-bearing,

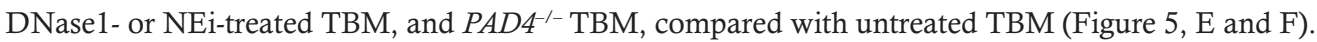

Together, these data indicate that primary tumors can induce metastasis-promoting NETs in the absence of sepsis and that NET-targeted therapies reduce both lung and liver metastasis.

\section{Discussion}

Inflammation and immune cell chemotaxis have been recognized as key players in cancer progression, and clinical and experimental evidence points to protumorigenic roles for neutrophils. Neutrophils are stimulated to release pathogen-trapping NETs as an antimicrobial mechanism in response to infectious stimuli (11). 
A
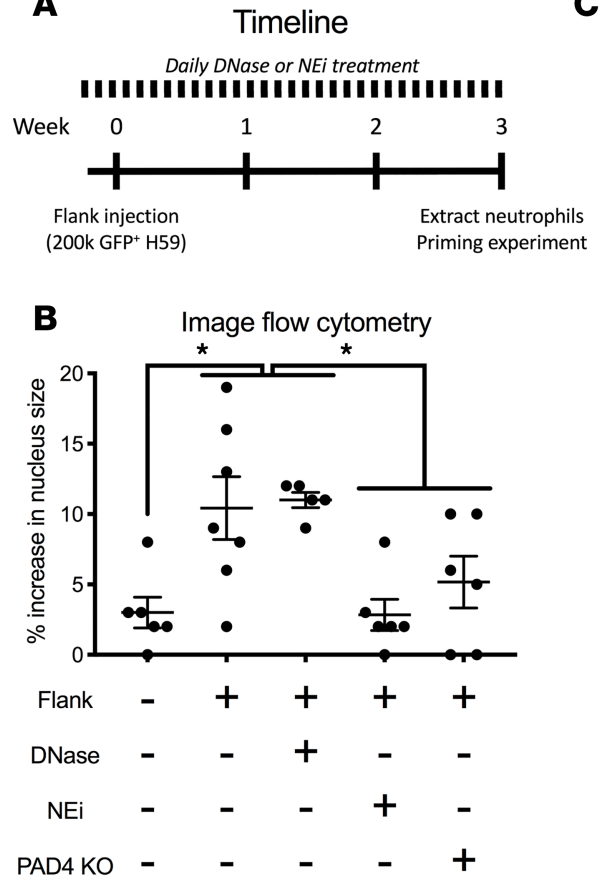

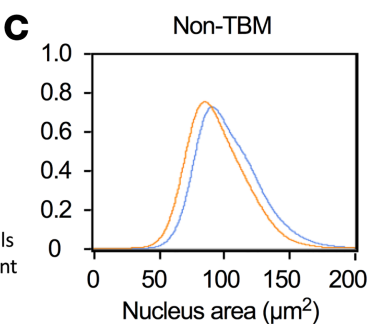

TBM + DNase

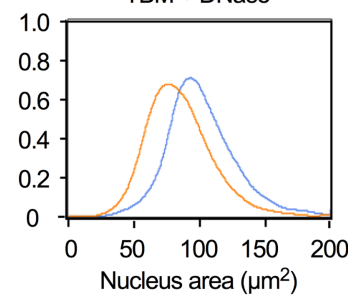

PAD4KO TBM

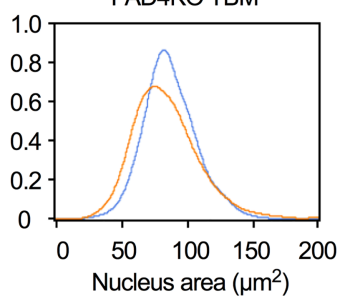

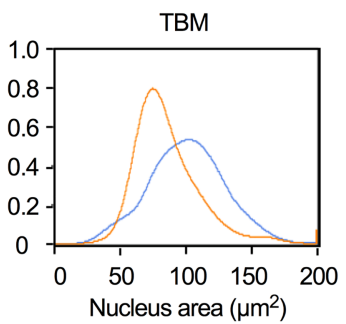

D
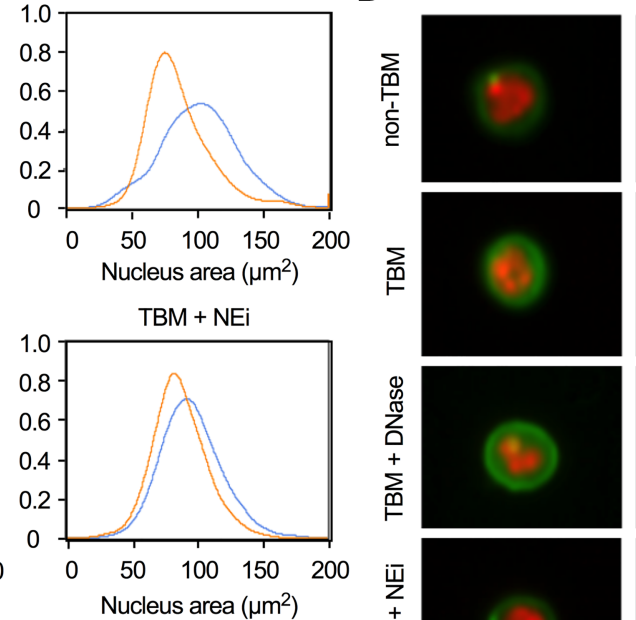

Baseline

PMA
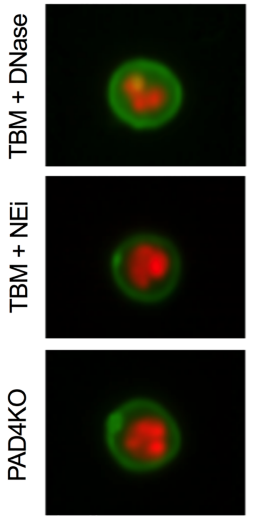

PMA-Stimulated
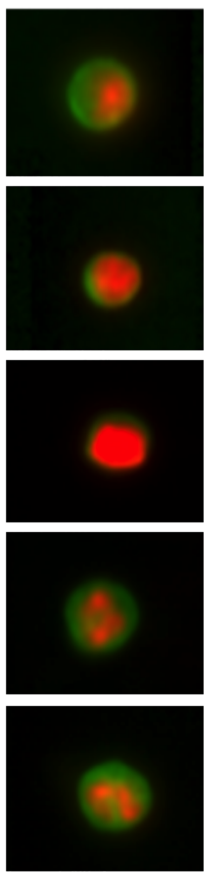

Figure 3. Neutrophils from TBM are more sensitized to NETosis compared with TBM treated with a NET inhibitor and in mice with inhibited NET formation. (A) Timeline of murine lung carcinoma priming experiment. (B) Dot graph of the mean ( \pm SEM) percent change in nuclear area following PMA stimulation for non-TBM $(n=6)$, TBM $(n=7)$, TBM treated with DNase1 $(n=6)$ or NEi $(n=6)$, and PAD4 ${ }^{-/-}$TBM $(n=6)$. One-way ANOVA was used to assess statistical significance. ${ }^{*} P<0.05$. (C) Representative histograms of the distribution of neutrophil nuclear area in the 5 group of mice from $\mathbf{B}$ are plotted at baseline (orange curve) and following PMA stimulation (blue curve). (D) Representative images of neutrophils from the 5 groups of mice from $\mathbf{B}$ and $\mathbf{C}$ at baseline and after stimulation. Neutrophils are stained with Ly6G (green); nuclei are stained with DAPI (orange). Magnification, 40×.

We were the first to our knowledge to show that NETs trap circulating tumor cells (CTCs) in the microvasculature, increasing tumor adhesion and promoting metastasis in a murine model of postoperative infection (15). Here, we describe that NET levels are elevated in lung, gastric, and esophageal cancer patients and that high levels of NETs are predictive of advanced disease. Furthermore, we find that cancer is an independent predictor of NET formation over other comorbidities. Inhibiting or degrading NETs leads to decreased lung and colon cancer adhesion and metastasis in a murine model, suggesting that NET-targeted therapies may limit metastasis.

Besides tumor stage, diabetes was also an independent predictor of elevated NET levels. This result is consistent with previous work showing that neutrophils from diabetic patients are primed for NETosis (39) and that high NET levels are correlated with type 2 diabetes (40). In addition, there was a trend toward higher NET levels in patients with high BMI, which is relevant, given that obesity is associated with chronic, low-grade inflammation that can modulate the TIME (reviewed in ref. 41) and facilitate breast cancer cell metastasis via increased neutrophil recruitment (42).

Several conditions that are known to induce extensive inflammation were shown to lead to an increase in NET levels in humans $(21-26,39,40,43)$. Here, we show that, in addition to those conditions, primary tumors alone - from multiple disease sites and in the absence of any infection - can induce NETs. This is not totally unexpected, since one of the hallmarks of cancer is inflammation (44). Moreover, a recent preclinical study has shown that metastatic breast cancer cells can induce metastasis-supporting NETs in mice, where treatment with DNase1-coated nanoparticles reduced lung metastasis (20). This is in line with our findings that DNase1 or NEi treatments decrease liver and lung metastasis. These seminal findings advocate for the development of clinical trials to determine whether NET-targeted therapies can reduce metastasis.

To date, there are no clinical trials to our knowledge to evaluate the effects of targeting NETs on cancer progression. There is only one Phase 1 pilot study assessing the effects of recombinant DNase 1 in patients with head and neck cancers treated with radiation therapy and chemotherapy 
A

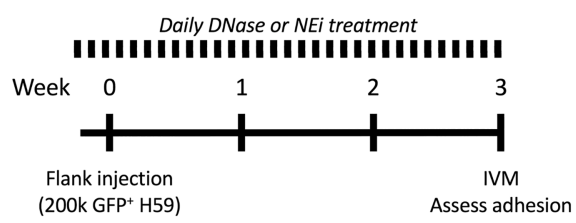

D IVM adhesion assay (MC38-GFP)

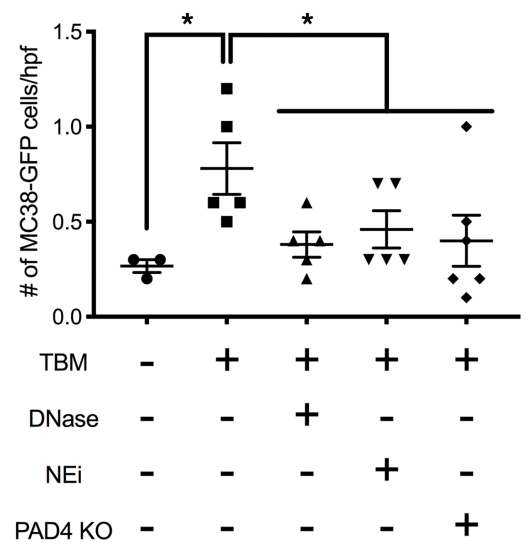

B IVM adhesion assay (H59-GFP)

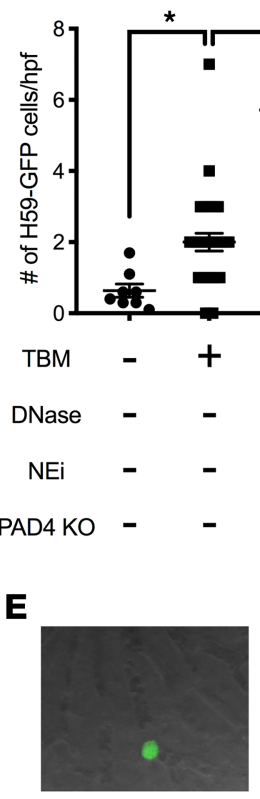

Non-TBM

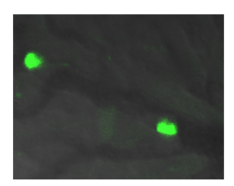

TBM

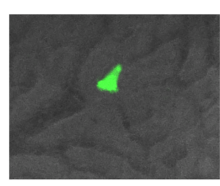

$\mathrm{TBM}+\mathrm{DNase}$

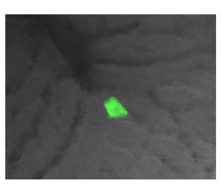

$\mathrm{TBM}+\mathrm{NEi}$

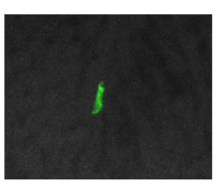

PAD4KO TBM

Figure 4. NET-deficient mice show reduced lung and colon cancer cell adhesion to the liver. (A) Timeline of murine intravital microscopy (IVM) experiment. (B) Mean ( \pm SEM) number of adherent lung carcinoma cells (H59-GFP) per field in the 5 groups of mice. (C) Representative fluorescence confocal microscopy images of IVM performed on the mice from B, showing H59-GFP cells in green; liver sinusoids are shown in blue (CD31-PE staining). (D) Mean $( \pm$ SEM) number of adherent colon carcinoma cells (MC38-GFP) per field in the 5 groups of mice. (E) Representative fluorescence confocal microscopy images of IVM performed on the mice from D, showing MC38-GFP cells in green; liver sinusoids are shown using bright field. For all panels: $n=3-6$ mice, with 10 fields per mouse. A Kruskal-Wallis test was used to calculate significance for $\mathbf{B}$ and $\mathbf{D}$ because the data were not normally distributed, as assessed by the Kolmogorov-Smirnov test. ${ }^{*} P<0.05$. Magnification, $20 \times$.

(NCT00536952; https://clinicaltrials.gov). Although not a goal of the study, it would be interesting to see if DNase1 treatment will delay cancer progression and metastasis.

Our study shows that inhibition of NETs via either NEi or using $P A D 4^{-1-}$ mice leads to a decrease in NET levels, which translates into less in vivo adhesion of circulating tumor cells to the liver sinusoids and a decrease in spontaneous lung and liver metastasis, a similar effect seen when DNase1 is administrated in those mice. Since NE and PAD4 are implicated in different NETosis pathways (ROS-dependent and ROS-independent) and seeing that DNase1 treatment does not have a larger effect as compared with NE and PAD4 inhibition, this indicates that NEi and $P A D 4^{-/-}$act on the same pathway.

Moreover, despite having a NET-mediated systemic effect that is promoting tumor metastasis, we found that the primary tumors of our nonseptic mouse models did not induce a massive systemic inflammatory signature, as seen in our septic mouse model (15). Indeed, we do not observe a massive deposition of NETs in the secondary organs (lungs and livers) of nonseptic TBM (Supplemental Figure 7), as was seen in the CLP mice (15). In addition, we are able to detect NETs in the primary tumor (flank) (Supplemental Figure 7A) but not in either of the metastatic sites (Supplemental Figure 7, A and B) of nonseptic TBM. Therefore, unlike in a septic mouse model (15), where we were able to observe NET deposition in the lungs and livers of CLP mice (by IVM and IHC), here - in nonseptic TBM - we were only able to detect NETs in circulation (by ELISA). In addition, circulating neutrophils are primed to NETose, as seen using imaging flow cytometry, another indication that NETosis is occurring in those mice. Therefore, primary tumor-induced NETs can promote tumor metastasis by trapping CTCs (15) or by other mechanisms such as promoting thrombosis (reviewed in ref. 45), for example.

Several preclinical studies have shown that NETs can induce tumor growth and metastasis. However, most of those studies were done in the context of a massive inflammation or infection, such as sepsis (15), surgical stress (21), and prolonged tobacco smoke (17). Here, we show that primary tumors are sufficient to induce metastasis-promoting NETs. Moreover, our study establishes circulating NET levels as a tumor-induced and prognostically significant biomarker. The emerging concept that a patient's immune 
A

Timeline

\section{Daily DNase or NEi treatment}

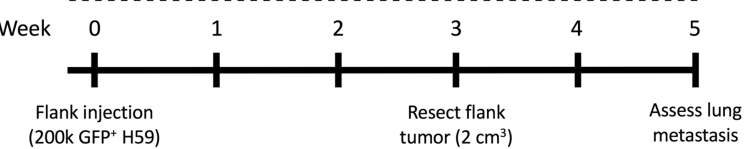

B

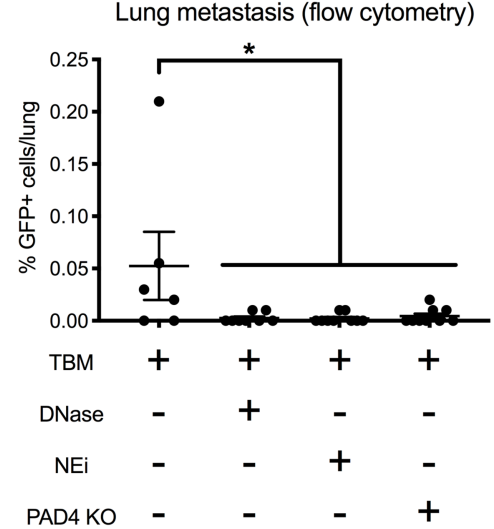

C

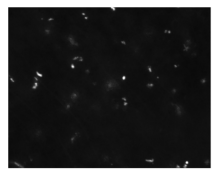

TBM

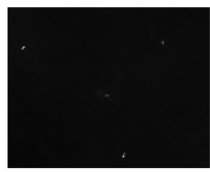

TBM + DNase

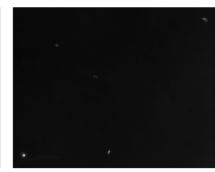

$\mathrm{TBM}+\mathrm{NEi}$

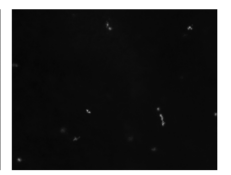

PAD4KO TBM

D

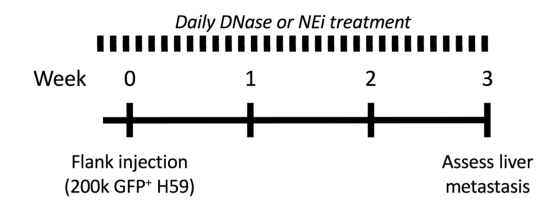

E

Liver metastasis (fluorescence microscopy)

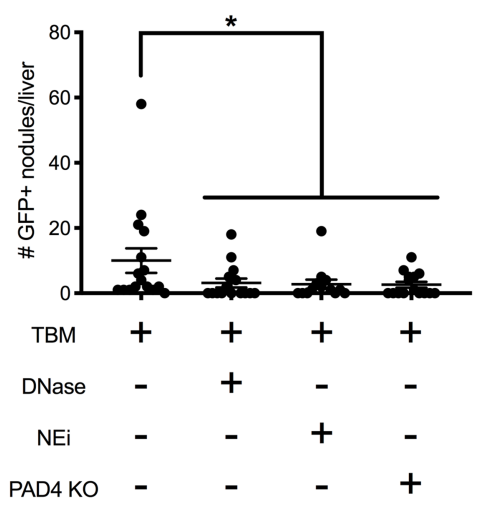

$\boldsymbol{F}$

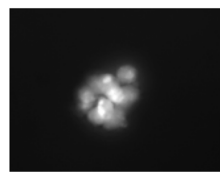

TBM

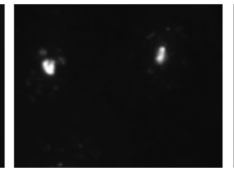

$\mathrm{TBM}+\mathrm{DNase}$

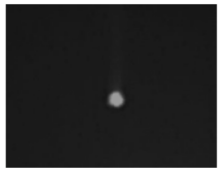

$\mathrm{TBM}+\mathrm{NEi}$

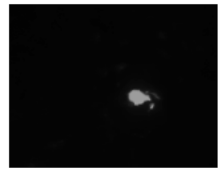

PAD4KO TBM

Figure 5. NET-deficient mice show reduced spontaneous lung and liver metastasis of lung carcinoma cells. (A) Timeline of the murine spontaneous liver and lung metastasis experiment with resection. (B) Mean ( \pm SEM) percentage of H59-GFP+ cells in the lungs of TBM $(n=6)$ and DNase1-treated $(n=6)$, NEi-treated $(n=9)$, and PAD4 ${ }^{-/-}(n=8)$ TBM. (C) Representative fluorescence microscopy images of spontaneous lung metastases in the 4 groups of mice from B. Magnification, 10×. (D) Timeline of the murine spontaneous liver metastasis experiment without resection. (E) Mean ( \pm SEM) number of H59-GFP+ cells in livers of TBM $(n=16)$ and DNase1-treated $(n=15)$, NEi-treated $(n=13)$, and PAD4 $4^{--}(n=15)$ TBM. (F) Representative fluorescence microscopy images of spontaneous liver metastases in the 4 groups of mice from $\mathbf{E}$. Magnification, 20x. A Kruskal-Wallis test was used to calculate significance for $\mathbf{B}$ and $\mathbf{E}$ because the data were not normally distributed, as assessed by the Kolmogorov-Smirnov test. ${ }^{*} P<0.05$.

state both systemically and within the developing cancer is a critical factor underlying response to immunotherapy further underpins the importance of our findings. Indeed, developing an understanding of how circulating NET levels impact response to treatment in cancer patients will be of critical importance to future studies. Both Sivelestat (NEi) and DNase1 have been tested and used for treating several respiratory conditions in humans at similar or higher concentrations; thus, safety and pharmacokinetics studies have already been performed $(46,47)$. This simplifies the process of initiating a phase II clinical trial in cancer patients with either agent. This study, therefore, advocates for the use of NET-based therapeutics in cancer treatment to limit metastasis from several malignancies.

In conclusion, with all recent publications linking elevated NET levels with the progression of several malignancies, such as breast (20) and colorectal (28) cancers, pancreatic ductal adenocarcinoma (29), ovarian metastasis (31), and - here - lung and gastroesophageal adenocarcinomas, it is interesting to see how successful NET-targeted therapies will be in clinical trials and how helpful NET-based biomarkers will be in predicting and prognosticating cancer progression and metastasis.

\section{Methods}

Model-building strategy for the multivariate analysis. The analysis was performed to identify whether NET absorbance is a significant independent predictor of disease progression (overall stage) and adjusting for important available confounders: age, sex, BMI, smoking, diabetes, and comorbidities. All confounders were chosen because they have been shown to induce NET release $(39,48,49)$. Multivariable logistic regression analyses were performed, and model assumptions were tested using graphical representations of 
residuals, residuals versus main predictor variable graphs, and residuals versus predicted variable graphs. Model fit was tested using the likelihood ratio test with nested models.

Clinical staging, instead of pathological, was used for esophagogastric adenocarcinomas, given the downstaging effects of chemotherapy (50-52), with $1 / 3$ patients receiving neoadjuvant chemotherapy and $1 / 5$ having inoperable disease.

Cell lines. Murine Lewis Lung carcinoma cell subline H59, expressing stable GFP (H59-GFP), was a gift of Pnina Brodt (53) and maintained in RPMI supplemented with 10\% FBS, $100 \mu \mathrm{g} / \mathrm{ml}$ penicillin/ streptomycin, and $300 \mu \mathrm{g} / \mathrm{ml}$ glutamine (Wisent Bioproducts). Murine colon carcinoma cell line MC38 was a gift of Nicole Beauchemin (54) and maintained in DMEM media supplemented with 10\% FBS and $100 \mu \mathrm{g} / \mathrm{ml}$ penicillin/streptomycin. MC38-GFP and MC38-RFP were engineered by transduction of MC38 with viral particles containing GFP and RFP, respectively, according to manufacturer's instructions (CMV-Luciferase[firefly]-2A-GFP[Puro] and CMV-Luciferase[firefly]-2A-RFP[Puro] from GenTarget).

Animals. C57BL/6 (Charles River Laboratories) and PAD4-KO (PAD4 $4^{--}$; gift of Alan Tsung, The Ohio State University Comprehensive Cancer Center, Columbus, Ohio, USA) male mice were used for all experiments at 7-10 weeks old. MC38-RFP and H59-GFP were injected s.c. into the flank of these mice, and tumor growth was monitored twice a week using a caliper. Mice were divided into untreated, daily i.m. injection of $2.5 \mathrm{mg} / \mathrm{kg}$ DNase1 (Roche Diagnostics), gavage of $2.2 \mathrm{mg} / \mathrm{kg}$ of Sivelestat (NEi, Abcam), and PAD4 $4^{--}$mice.

Blood collection and processing. Human blood was obtained from consented patients and healthy volunteers as per lung (MUHC IRB 2014-1119) and esophageal/gastric (MUHC IRB 2007-856) biobanks. Mouse blood was collected by heart puncture from anesthetized mice. Human and mouse blood was collected in heparinized tubes and centrifuged at $500 \mathrm{~g}$ for 10 minutes at $4^{\circ} \mathrm{C}$. Plasma was collected and stored at $-80^{\circ} \mathrm{C}$.

NETS ELISA. Immulon 4HBX 96-well plates (Thermo Fisher Scientific) were coated with mouse or human MPO antibody (clone 266-6K1, 1:20, Hycult Biotechnology) overnight at $4^{\circ} \mathrm{C}$. Wells were washed with PBS (wash step) and blocked with 1\% BSA (Wisent Bioproducts) for 1 hour at room temperature (RT). Following a wash step, plasma was mixed with dsDNA-POD antibody (1:40, Cell death detection ELISA plus kit, Roche Diagnostics) and added to wells for 2 hours shaking at RT. Following a wash step, ABTS (Roche Diagnostics) was added for 30 minutes at $37^{\circ} \mathrm{C}$, and the plate was read at $405 \mathrm{~nm}$.

Neutrophil isolation from human peripheral blood. Human neutrophil isolation is described in ref. 55.

Neutrophil isolation from mouse peripheral blood. Murine neutrophil isolation was similar to human neutrophil isolation, but neutrophils were stained with Ly6G prior to use.

Neutrophil isolation from mouse BM. Mouse neutrophil isolation is described in ref. 56.

Imaging flow cytometry. Peripheral blood neutrophils were isolated from mice with flank tumors the sizes of $1.5-2 \mathrm{~cm}^{3}$ and fixed with $2 \%$ PFA. They were then stained with DAPI $(1: 2,000$, Thermo Fisher Scientific) and Ly6G-FITC (clone 1A8, 1:200, Thermo Fisher Scientific). Imaging flow cytometry was performed on an Amnis ImageStream MarkII (MilliporeSigma).

IVM. Mice with H59-GFP or MC38-RFP tumors the sizes of $1.5-2 \mathrm{~cm}^{3}$ were injected intrasplenically with 3 $\times 10^{4}$ H59-GFP or MC38-GFP cancer cells, respectively, and adhesion to liver sinusoids was visualized after 10 minutes, as described in ref. 15, using an LSM-780 confocal microscope (Carl Zeiss).

Flow cytometry. Lungs were excised from previously resected mice and minced using scissors, to which $10 \mathrm{ml}$ of Liberase (MilliporeSigma) in L-15 media (Wisent Bioproducts) were added and left shaking at $37^{\circ} \mathrm{C}$ for 30 minutes. Homogenates were vigorously mixed and incubated for another $30-$ minute shaking at $37^{\circ} \mathrm{C}$. Homogenates were strained through $70-\mu \mathrm{M}$ filters, centrifuged at $480 \mathrm{~g}$ for 5 minutes, resuspended in PBS, and analyzed by flow cytometry for H59-GFP ${ }^{+}$using FACScan and CellQuest Software for analysis (BD Biosciences).

Spontaneous liver metastasis from H59-GFP flank-injected mice. Livers from H59-GFP flank-injected mice were excised when the flank tumor size reached $2 \mathrm{~cm}^{3}$ for nonresected mice and 2 weeks after resection for resected mice; hepatic nodules were counted under a fluorescent microscope.

Western blot. Flank tumors, livers, and lungs from C57BL/6 TBM were homogenized, and protein was extracted using RIPA buffer (Thermo Fisher Scientific) as per manufacturer's instructions. Protein concentration was assessed using a BCA assay (Bio-Rad).

Cell lysates $(250 \mu \mathrm{g})$ were loaded on a $12 \%$ SDS-PAGE and ran at $100 \mathrm{~V}$ for 90 minutes at RT. Proteins were transferred onto a nitrocellulose membrane (Pall Corporation) at $90 \mathrm{~V}$ for 90 minutes in the cold room. Membranes were probed with H3Cit (Abcam, catalog 5103, 1:1000) and $\beta$-actin (MilliporeSigma, catalog A5441, 1:10,000) diluted in TBS-Tween (0.1\%) for 1 hour at RT. Membranes were washed 3 times, 5 minutes each, in TBST and then probed using HRP anti-rabbit (polyclonal, catalog 111-035-144, 1:3,000) 
and HRP anti-mouse (polyclonal, catalog 115-035-003, 1:2,000) (both from Jackson ImmunoResearch) for $\mathrm{H} 3 \mathrm{Cit}$ and $\beta$-actin, respectively. Membranes were incubated with Clarity Western ECL substrate (Bio-Rad) for 5 minutes prior to imaging using the LAS4000 ImageQuant (Perkin Elmer).

Immunofluorescence. Livers and lungs from C57BL/6 mice were fixed with formalin for 48 hours and then paraffin embedded. FFPE blocks were then sectioned and stained at the histopathology platform of the Research Institute-MUHC (RI-MUHC). H3Cit (Abcam, catalog 5103, 1:100) and Ly6G-AF647 (clone 1A8, BioLegend, 1:200) were used with a PE-conjugated anti-rabbit antibody (polyclonal, catalog P-2771MP, Invitrogen) as a secondary for H3Cit. Images were acquired on a LSM780 laser scanning confocal microscope (Zeiss) using an EC Plan-Neofluar 10×/0.30 lens.

Statistics. A Kolmogorov-Smirnov test was performed for each figure to assess if our data are normally distributed. For normally distributed data,2-tailed $t$ tests and 1-way ANOVA were performed to compare mean values between different groups. A Kruskal-Wallis nonparametric test was used to assess statistical differences between the different groups. In all cases, a $P$ value less than 0.05 was considered significant.

Study approval. All mice experiments were carried out in strict accordance with the recommendations of the Canadian Council on Animal Care (CCAC) Guide to the Care and Use of Experimental Animals' and under the conditions and procedures approved by the Animal Care Committee of McGill University (AUP 7724). All clinical work was performed under the considerations and procedures approved by the MUHC Research Ethics Board (REB) (projects 2007-856 and 2014-1119). Written informed consent was received from participants prior to inclusion in the study.

\section{Author contributions}

RFR and JGM conducted all the experiments, acquired and analyzed the data, and wrote the paper. IN conducted the multivariate analysis. FB helped conduct the in vivo experiments. BG helped conduct the in vitro experiments. SR, DQ, LW, VS, NB, JCL, and LEF provided input and guidance. JDS planned, oversaw, and guided the project.

\section{Acknowledgments}

We would like to thank Min Fu (manager molecular imaging platform), Marie Helene Lacombe (manager immunophenotyping platform), and Fazila Chaouli (manager histopathology platforms), as well as all the staff members of those core facilities for their help in planning, operating, and analyzing all of our experiments. Funding for this project came from the Canadian Institutes of Health Research (CIHR) (to LF), the American Association for Thoracic Surgery (AATS) (to JDS), and Fonds de Recherche en Santé du Québec (FRQS) (to JDS).

Address correspondence to: Jonathan D. Spicer, Montreal General Hospital, Room L8-505-1650 Cedar Avenue, Montreal, Québec, Canada H3G 1A4. Phone: 514.934.1934 ext. 43050; Email: jonathan.spicer@mcgill.ca.

1. Binnewies M, et al. Understanding the tumor immune microenvironment (TIME) for effective therapy. Nat Med. 2018;24(5):541-550.

2. Sharma P, Allison JP. The future of immune checkpoint therapy. Science. 2015;348(6230):56-61.

3. Sharon E, Streicher H, Goncalves P, Chen HX. Immune checkpoint inhibitors in clinical trials. Chin J Cancer. 2014;33(9):434-444.

4. McDonald B, Spicer J, Giannais B, Fallavollita L, Brodt P, Ferri LE. Systemic inflammation increases cancer cell adhesion to hepatic sinusoids by neutrophil mediated mechanisms. Int J Cancer. 2009;125(6):1298-1305.

5. Spicer JD, et al. Neutrophils promote liver metastasis via Mac-1-mediated interactions with circulating tumor cells. Cancer Res. 2012;72(16):3919-3927.

6. Singhal S, et al. Origin and Role of a Subset of Tumor-Associated Neutrophils with Antigen-Presenting Cell Features in Early-Stage Human Lung Cancer. Cancer Cell. 2016;30(1):120-135.

7. Wculek SK, Malanchi I. Neutrophils support lung colonization of metastasis-initiating breast cancer cells. Nature. 2015;528(7582):413-417.

8. Eruslanov EB, et al. Tumor-associated neutrophils stimulate T cell responses in early-stage human lung cancer. J Clin Invest. 2014;124(12):5466-5480.

9. Tazzyman S, Lewis CE, Murdoch C. Neutrophils: key mediators of tumour angiogenesis. Int J Exp Pathol. 2009;90(3):222-231.

10. Tüting T, de Visser KE. CANCER. How neutrophils promote metastasis. Science. 2016;352(6282):145-146.

11. Brinkmann V, et al. Neutrophil extracellular traps kill bacteria. Science. 2004;303(5663):1532-1535.

12. Honda M, Kubes P. Neutrophils and neutrophil extracellular traps in the liver and gastrointestinal system. Nat Rev Gastroenterol Hepatol. 2018;15(4):206-221.

13. Kolaczkowska E, et al. Molecular mechanisms of NET formation and degradation revealed by intravital imaging in the liver 
vasculature. Nat Commun. 2015;6:6673.

14. Jorch SK, Kubes P. An emerging role for neutrophil extracellular traps in noninfectious disease. Nat Med. 2017;23(3):279-287.

15. Cools-Lartigue J, et al. Neutrophil extracellular traps sequester circulating tumor cells and promote metastasis. J Clin Invest. 2013;123(8):3446-3458.

16. Cools-Lartigue J, Spicer J, Najmeh S, Ferri L. Neutrophil extracellular traps in cancer progression. Cell Mol Life Sci. 2014;71(21):4179-4194.

17. Albrengues J, et al. Neutrophil extracellular traps produced during inflammation awaken dormant cancer cells in mice. Science. 2018;361(6409):eaao4227.

18. Demers M, et al. Cancers predispose neutrophils to release extracellular DNA traps that contribute to cancer-associated thrombosis. Proc Natl Acad Sci USA. 2012;109(32):13076-13081.

19. Demers M, et al. Priming of neutrophils toward NETosis promotes tumor growth. Oncoimmunology. 2016;5(5):e1134073.

20. Park J, et al. Cancer cells induce metastasis-supporting neutrophil extracellular DNA traps. Sci Transl Med. 2016;8(361):361ra138

21. Tohme S, et al. Neutrophil Extracellular Traps Promote the Development and Progression of Liver Metastases after Surgical Stress. Cancer Res. 2016;76(6):1367-1380.

22. Kessenbrock K, et al. Netting neutrophils in autoimmune small-vessel vasculitis. Nat Med. 2009;15(6):623-625.

23. Sur Chowdhury C, Giaglis S, Walker UA, Buser A, Hahn S, Hasler P. Enhanced neutrophil extracellular trap generation in rheumatoid arthritis: analysis of underlying signal transduction pathways and potential diagnostic utility. Arthritis Res Ther. 2014;16(3):R122.

24. Caudrillier A, et al. Platelets induce neutrophil extracellular traps in transfusion-related acute lung injury. J Clin Invest. 2012;122(7):2661-2671.

25. Fuchs TA, Brill A, Wagner DD. Neutrophil extracellular trap (NET) impact on deep vein thrombosis. Arterioscler Thromb Vasc Biol. 2012;32(8):1777-1783.

26. Arai Y, et al. Serum neutrophil extracellular trap levels predict thrombotic microangiopathy after allogeneic stem cell transplantation. Biol Blood Marrow Transplant. 2013;19(12):1683-1689.

27. Erpenbeck L, Schön MP. Neutrophil extracellular traps: protagonists of cancer progression? Oncogene. 2017;36(18):2483-2490.

28. Richardson JJR, Hendrickse C, Gao-Smith F, Thickett DR. Neutrophil Extracellular Trap Production in Patients with Colorectal Cancer In Vitro. Int J Inflam. 2017;2017:4915062.

29. Jin W, et al. Tumor-Infiltrating NETs Predict Postsurgical Survival in Patients with Pancreatic Ductal Adenocarcinoma. Ann Surg Oncol. 2019;26(2):635-643.

30. Thålin $\mathrm{C}$, et al. Citrullinated histone $\mathrm{H} 3$ as a novel prognostic blood marker in patients with advanced cancer. PLoS ONE. 2018;13(1):e0191231.

31. Lee W, Ko SY, Mohamed MS, Kenny HA, Lengyel E, Naora H. Neutrophils facilitate ovarian cancer premetastatic niche formation in the omentum. J Exp Med. 2019;216(1):176-194.

32. Najmeh S, et al. Neutrophil extracellular traps sequester circulating tumor cells via $\beta 1$-integrin mediated interactions. Int J Cancer. 2017;140(10):2321-2330.

33. Hemmers S, Teijaro JR, Arandjelovic S, Mowen KA. PAD4-mediated neutrophil extracellular trap formation is not required for immunity against influenza infection. PLOS ONE. 2011;6(7):e22043.

34. Li P, Li M, Lindberg MR, Kennett MJ, Xiong N, Wang Y. PAD4 is essential for antibacterial innate immunity mediated by neutrophil extracellular traps. J Exp Med. 2010;207(9):1853-1862.

35. Tatsiy O, McDonald PP. Physiological Stimuli Induce PAD4-Dependent, ROS-Independent NETosis, With Early and Late Events Controlled by Discrete Signaling Pathways. Front Immunol. 2018;9:2036.

36. Zhao W, Fogg DK, Kaplan MJ. A novel image-based quantitative method for the characterization of NETosis. J Immunol Methods. 2015;423:104-110.

37. McDonald B, Urrutia R, Yipp BG, Jenne CN, Kubes P. Intravascular neutrophil extracellular traps capture bacteria from the bloodstream during sepsis. Cell Host Microbe. 2012;12(3):324-333.

38. Brodt P. Characterization of two highly metastatic variants of Lewis lung carcinoma with different organ specificities. Cancer Res. 1986;46(5):2442-2448.

39. Wong SL, et al. Diabetes primes neutrophils to undergo NETosis, which impairs wound healing. Nat Med. 2015;21(7):815-819.

40. Menegazzo L, et al. NETosis is induced by high glucose and associated with type 2 diabetes. Acta Diabetol. 2015;52(3):497-503.

41. Olson OC, Quail DF, Joyce JA. Obesity and the tumor microenvironment. Science. 2017;358(6367):1130-1131.

42. Quail DF, et al. Obesity alters the lung myeloid cell landscape to enhance breast cancer metastasis through IL5 and GM-CSF. Nat Cell Biol. 2017;19(8):974-987.

43. Itagaki $\mathrm{K}$, et al. Mitochondrial DNA released by trauma induces neutrophil extracellular traps. PLoS ONE. 2015;10(3):e0120549.

44. Hanahan D, Weinberg RA. Hallmarks of cancer: the next generation. Cell. 2011;144(5):646-674.

45. Cedervall J, Olsson AK. Immunity Gone Astray - NETs in Cancer. Trends Cancer. 2016;2(11):633-634.

46. Kido T, et al. Efficacy of early sivelestat administration on acute lung injury and acute respiratory distress syndrome. Respirology. 2017;22(4):708-713

47. Shak S, Capon DJ, Hellmiss R, Marsters SA, Baker CL. Recombinant human DNase I reduces the viscosity of cystic fibrosis sputum. Proc Natl Acad Sci USA. 1990;87(23):9188-9192.

48. Qiu SL, et al. Neutrophil extracellular traps induced by cigarette smoke activate plasmacytoid dendritic cells. Thorax. 2017;72(12):1084-1093

49. Wang H, et al. Obesity-induced Endothelial Dysfunction is Prevented by Neutrophil Extracellular Trap Inhibition. Sci Rep. 2018;8(1):4881.

50. Andriessen MJ, van der Peet DL, Cuesta MA. Effect of neoadjuvant chemotherapy on circumferential margin positivity and its impact on prognosis in patients with resectable oesophageal cancer. Br J Surg. 2008; 95(2): 191-194.

51. Matsuyama J, et al. The effect of neoadjuvant chemotherapy on lymph node micrometastases in squamous cell carcinomas of 
the thoracic esophagus. Surgery. 2007;141(5):570-580.

52. Sujendran V, Wheeler J, Baron R, Warren BF, Maynard N. Effect of neoadjuvant chemotherapy on circumferential margin positivity and its impact on prognosis in patients with resectable oesophageal cancer. Br J Surg. 2008;95(2):191-194

53. Auguste P, Fallavollita L, Wang N, Burnier J, Bikfalvi A, Brodt P. The host inflammatory response promotes liver metastasis by increasing tumor cell arrest and extravasation. Am J Pathol. 2007;170(5):1781-1792.

54. Arabzadeh A, et al. Host-related carcinoembryonic antigen cell adhesion molecule 1 promotes metastasis of colorectal cancer Oncogene. 2013;32(7):849-860.

55. Najmeh S, Cools-Lartigue J, Giannias B, Spicer J, Ferri LE. Simplified Human Neutrophil Extracellular Traps (NETs) Isolation and Handling. J Vis Exp. 2015; e52687.

56. Mócsai A, Zhang H, Jakus Z, Kitaura J, Kawakami T, Lowell CA. G-protein-coupled receptor signaling in Syk-deficient neutrophils and mast cells. Blood. 2003;101(10):4155-4163. 